\title{
REPRESENTACIONES SOCIALES DE LA CORRUPCIÓN Y LA VIOLENCIA Y SU RELACIÓN CON LA REFLEXIÓN SOCIO MORAL EN JÓVENES DE ZONAS URBANAS Y RURALES DE DIVERSAS REGIONES DEL PERÚ
}

Social representations of corruption and violence and its relationship with social moral reflection in young urban and rural areas of different regions of Peru

Carlos Arenas I. ${ }^{1}$, María Matalinares C., Víctor Montero L., Eli Malvaceda E., Israel Rivera P., Elsa Sánchez Y.

Universidad Nacional Mayor de San Marcos, Lima, Perú

(ReCibido el 26/05/2011 - AcEPtAdo el 09/06/2011)

\begin{abstract}
RESUMEN
La investigación identificó las representaciones sociales sobre la corrupción y la violencia y su relación con los niveles de reflexión socio-moral de los jóvenes de procedencia urbana y rural del país. Se evaluó a 268 jóvenes de zonas urbanas y rurales, de ambos sexos, cuyas edades fluctuaban entre 18 y 25 años, se desarrolló grupos focales a fin de identificar las representaciones sociales de la corrupción y la violencia y se aplicó el cuestionario de reflexión socio-moral propuesto Gibbs, Arnold y Morgan y adaptado por Grimaldo (2002). No se halló relación significativa entre la reflexión socio-moral y la representación social de la corrupción y la violencia. Existe un desarrollo moral mayormente ubicado en la concordia interpersonal, aunque un tercio de la población se ubica en una orientación instrumental relativista y el nivel de desarrollo moral alcanzado por los jóvenes corresponde al convencional y preconvencional. Los niveles de reflexión socio-moral en Pucallpa se ubicaría en el Estadio 2 (orientación instrumental relativista) mientras que el resto de regiones evaluadas el Estadio 3 (orientación hacia la concordia interpersonal) sería el predominante. La corrupción sería una práctica social de adaptación que permite la orientación a la concordia interpersonal, en muchos casos se tiene una visión pesimista sobre la posibilidad de eliminarlas. La representación social de la violencia tendría relación directa con los actos de corrupción, en el sentido de dar mayor valor al beneficio individual e inmediato que al bienestar social o colectivo.
\end{abstract}

Palabras clave: Reflexión socio-moral, representaciones sociales, corrupción, violencia, jóvenes.

\begin{abstract}
Research identified the social representations of corruption and violence and its relationship to levels of socio-moral reflection of young people in urban areas and rural country. We evaluated to 268 young people from urban and rural areas, of both sexes, aged between 18 and 25 years, developing focus groups to identify the social representations of corruption and
\end{abstract}

1 Profesor Principal de la Facultad de Psicología de la UNMSM. E-maill: postgradopsi@hotmail.com 
violence and a questionnaire of socio-reflection proposed moral Gibbs, Arnold and Morgan and adapted by Grimaldo (2002). No significant relationship was found between socio-moral reflection and social representation of corruption and violence. There is a moral development mostly located in the interpersonal harmony, while a third of the population is located in an instrumental relativist orientation and moral development level reached by youth for the preconventional and conventional. The levels of socio-moral reflection in Pucallpa be located in the Stage 2 (instrumental relativist orientation) while the other regions evaluated the Stage 3 (interpersonal harmony orientation) would be predominant. Corruption is a social practice of adaptation that allows orientation to interpersonal harmony, often have a pessimistic view about the possibility of eliminating them. The social representation of violence would be directly related to corruption, in the sense of giving greater value to the individual and immediate benefit to social welfare or collective.

Keywords: Socio-moral reflection, social representations, corruption, violence, youth.

\section{INTRODUCCIÓN}

La presencia de fenómenos sociales en el Perú, tales como la corrupción y la violencia, no son novedosos. La extensión y profundidad se han hecho cada vez más evidentes, aunque organismos internacionales como Transparencia Internacional ubican al Perú en el ranking de percepción de corrupción el país ha caído tres puestos -del 75 al 78-, sigue teniendo casi el mismo puntaje que hace cinco años: estamos a mitad de tabla, pero tampoco hemos empeorado. Los 3,5 puntos obtenidos por el Perú este año lo ubican más cerca de Venezuela, que con dos puntos está en el último lugar del continente, pero lo alejan más de Chile, que tiene el doble de puntos y que mejoró cuatro posiciones esta vez. Lo mismo puede decirse de la violencia y la conflictividad presente en la cotidianidad del peruano.

Por ello, desde la psicología el abordaje a esta problemática social se hace necesario, para la presente investigación se considera pertinente el uso de la teoría de las representaciones sociales, en los temas de representaciones sociales y desarrollo moral. En nuestro país son múltiples los estudios sobre ambos temas: Seguín, Chiappo, Saco, Tueros, Arenas, Montero, Peña, Nizama, Gorriti, Mc Gregor $(2008,2009)$. Asimismo, existen un conjunto de investigaciones como los de María A. Cánepa y otros (1993) sobre como la violencia está presente en la juventud peruana en sus esquinas, rincones y pasadizos

Autores como Moscovici (1965), Baro (1982), Jodelet (1989), Parker (1988) han dado significativos aportes. Baró (1982) dice: "Una sociedad mantiene su unidad debido a la existencia de una conciencia colectiva. La conciencia colectiva consiste en un saber normativo, común a los miembros de una sociedad".

La corrupción es una práctica instrumental, esto es, quienes incurren en ella la ejercitan como un medio para lograr algún provecho personal o corporativo, violando las normas de las instituciones y lesionando el derecho de otras personas.

Plantearse la pregunta sobre las condiciones que promueven los actos de corrupción en una sociedad. Una serie de circunstancias y actitudes confluyen para favorecer la degradación moral y política de las instituciones. La concentración del poder político en pocas manos, la ausencia de fiscalización efectiva desde los fueros del Estado y la sociedad civil contribuyen a la constitución de situaciones de impunidad. 
Partimos de señalar, en relación a la corrupción, que según Fernández Dols, J.M. (1995), podría definirse como una manipulación o transgresión encubierta de las normas que rigen una organización racional, con vistas a lograr un beneficio privado. Todo el mundo cree entender cuando se habla de corrupción, pero tras el consenso existen graves malentendidos porque una de las características de la corrupción es su opacidad, su cuidado de las apariencias, su "discreto encanto", hay siempre personas "dispuestas" a corromperse, lo que determina la mayor incidencia de este tipo de problemas es, desde la lógica psicosocial, el tipo de situación en el que se ven inmersos los individuos.

Para el psicólogo social no hay culpables ni individuales ni colectivos (de señalar a los primeros ya viven jurados, jueces y fiscales; de señalar a los segundos algunos peligrosos visionarios y sus seguidores), para un psicólogo social sólo hay circunstancias colectivas que dan lugar a dichos actos.

Dentro de los estudios sobre representaciones sociales de la corrupción y la violencia tenemos algunas investigaciones importantes que repasaremos a continuación:

En el plano internacional, Clemente (1986) estudió las características psicológicas diferentes en personas delincuentes y no delincuentes, infractores de la ley que fueron estudiadas en relación a su inteligencia, locus de control, autoestima, personalidad y actitudes, estas personas obtuvieron puntajes similares en locus de control, autoestima, neuroticismo, introversión, dogmatismo, inteligencia, solo se diferenciaron en que las encarceladas o infractoras de la ley tienen menor inteligencia social o menos capacidad para desenvolverse en el ambiente social, es decir resalta la importancia de estas habilidades para prevenir delitos.

Boniolo (2009) en su trabajo sobre "Tramas corruptas vs relaciones ocasionales de corrupción: prácticas y representaciones sociales según clase social”, el cual fue realizado en Argentina. Así, los significados compartidos de las categorías emergen como elementos interpretativos de las prácticas que se construyen en la interacción entre los grupos sociales y las personas que lo integran. Las prácticas corruptas son concebidas en términos de intercambios ilegales o ilegítimos al margen de la ley y en perjuicio del bienestar social.

Asimismo, encontró que los significados e interpretaciones están impregnados del sistema de valores sociales y culturales, llevando implícita o explícitamente una valoración de la situación. Las ideas sobre la corrupción son concebidas como una trasgresión ética que atenta contra el bienestar social y tiene un alto contenido valorativo negativo.

Según el mismo autor (2009), la representación social deviene en un modelo o contramodelo de comportamiento como muestran los relatos. El concepto reconocido por los entrevistados, y definido en sus relatos, aparece como un modo de práctica social instaurada en la cultura social argentina.

Otro estudio es el de Borjas y Monasterio (2007), sobre "Representaciones sociales de la ética empresarial en Venezuela", donde se concluye que: la interpretación de la representación social de la ética empresarial en el contexto venezolano es producto de los conceptos, afirmaciones y percepciones que emergieron de los actores en la cotidianidad de la práctica empresarial. En este sentido, la representación social de la ética empresarial está soportada por una concepción de "lo que sé" y de "lo que creo". 
En cuanto a las percepciones de los problemas éticos, se siguen las ideas de Jodelet (2004), quien señala que las representaciones sociales más fuertes dentro de la sociedad parten con un planteamiento de creencias a través de la noción de agrupación de los sujetos en su pensamiento como una realidad actual de las sociedades. Se agrega información en cuanto al anclaje, mediante el cual lo no familiar o remoto es asimilado en las categorías de la cognición cotidiana y la objetivación que hace que las representaciones se proyecten en el mundo. El uso de la teoría de las representaciones sociales permite la comprensión de un evento que está en el colectivo de una sociedad: la representación social de la ética empresarial.

En relación a la violencia, según la Organización Mundial de la Salud (OMS), año a año en el mundo, más de 1,6 millones de personas pierden la vida en forma violenta y muchas otras sufren lesiones no mortales como resultado de la violencia autoinfligida, interpersonal o colectiva (OMS, 2003: 3 y ss.). Conflictos cada vez más complejos van desdibujando la impresión que produce la muerte, la hacen cotidiana y, de este modo, se normalizan estos hechos que pasan a ser parte de nuestra vida diaria.

\section{Violencia y Agresión}

Según la Real Academia Española (RAE, 2001), violencia es la cualidad de violento, acción y efecto de violentar o violentarse; asimismo, es aquello que está fuera de su natural estado situación o modo, que obra con ímpetu o fuerza. De otro lado, agresión es definida como el acto de acometer a alguien para matarlo, herirlo o hacerle daño. Se entiende así que la agresión es una expresión extrema de la violencia, en la cual se atenta contra otra persona y que, además, es intencional, ya que se constituye como un acto para hacer daño.

Para conocer cuáles son las representaciones sociales que se generan ante la violencia, resulta importante conocer acerca de la intencionalidad y valoración de esta. Lesionar a otra persona no constituye un acto de agresión per se, lo será siempre y cuando tenga el carácter de intencionalidad. Si bien establecer que existe o no intención resulta muy difícil, consideramos que en parte se esclarecerá a la luz de las consecuencias (finalidad) del acto, si este favorece o no, directa o indirectamente, a la persona que lo ejecutó, lo cual hace que sea o no una agresión, tal como señala Moreno:

Hay cientos de actos en los que se aplica un exceso de fuerza, y que son considerados lícitos, correctos y necesarios (empujar a un niño que va a ser atropellado, sacar una muela, abandonar la casa materna, etc.). Pero la mayor parte de actos violentos son considerados como no necesarios por quienes los sufren y se interpretan como algo negativo atribuible a la voluntad de quien aplica la fuerza. En estos casos hablamos de agresión: empujar violentamente a un niño cuando nos pregunta insistentemente algo, sacar una muela en una sesión de tortura o abandonar a su suerte a unos padres enfermos y desvalidos (Moreno, 2001: 3).

Todo acto violento lleva consigo las razones por las cuales se realiza, sean o no intencionales, lo cual es definido por su valoración social, puesto que mientras algunas razones son justificadas, otras no. Tanto la justificación como la valoración de la violencia 
dependen a su vez de los valores imperantes en la sociedad. En este sentido se deben recordar las palabras de Paulo Freire:

Una vez establecida la relación opresora; está instaurada la violencia. De ahí que, en la historia esta jamás haya sido iniciada por los oprimidos. ¡Cómo podrían los oprimidos iniciar la violencia si ellos son el resultado de una violencia! ¿Cómo podrían ser los promotores de algo que, al instaurarse objetivamente, los constituye?

... No existirían los oprimidos si no existiera una relación de violencia que los conforme como violentados, en una situación objetiva de opresión (2005: 56).

\section{Presupuestos de la violencia}

Se consideran algunos presupuestos de la violencia (Martín-Baró, 2003: 80 y ss.), tomados a modo de variables para su relación con las representaciones sociales.

Diversidad de la violencia. Consideramos que las representaciones sociales que se formen ante la violencia dependerá del tipo de violencia que se esté analizando, en ese sentido se ha de señalar que la violencia se presenta en diferentes formas y tipos, tales como la autoinflingida, interpersonal y colectivo-estructural, cada una con distintos subtipos cuya naturaleza es diferente, ya que puede ser física, psicológica, sexual o por negligencia, abandono u omisión (OMS, 2003: 6), lo que significa que no se pueden generalizar los actos violentos como si fueran iguales.

Carácter histórico de la violencia. Debido a este carácter es imposible entender la violencia fuera del contexto y el tiempo en los cuales se produce. Cada cultura es diferente, lo que hace que el acto de violencia también lo sea. Esto implica que el estudio de la violencia no podría realizarse con solo analizar el hecho o el acto concreto, sino que también importan sus antecedentes, predisponentes y desencadenantes, al igual que sus efectos en el corto y el largo plazo.

Incertidumbre de sus límites. De otro lado, la incertidumbre de los límites de la violencia, nos hace recapacitar, hasta qué punto la persona se encuentra en medio de una situación violenta, así un acto violento lleva usualmente a otro como efecto sobre quien lo recibe y puede transmitirse a otras personas, lo que se denomina la espiral de la violencia, pues esta se incrementa tanto cualitativa como cuantitativamente, lo que hace imposible establecer sus límites.

\section{Componentes del acto violento}

Otro de los puntos importantes en el tema de la violencia consiste en analizar cada uno de los componentes que hacen posible el acto violento, de este modo y siguiendo una vez más a Martín-Baró (2003: 83), se señalan los siguientes componentes del acto violento.

Estructura formal del acto. Es la forma en la cual se ejecuta el acto, su operacionalización. Conociendo la formalidad del acto como una totalidad de sentido se podrá señalar si fue un acto de violencia o de agresión, asimismo se distinguirá entre la violencia final u hostil 
y la violencia instrumental (Myers, 2003: 385). La primera se refiere al acto de violencia realizado por sí mismo, esto es, el acto buscado como un fin; por ejemplo, el asesinato de un campesino acusado de terrorista que constituye el fin en sí mismo. El segundo es un acto realizado como un medio para lograr un objetivo diferente.

Forma personal del acto. Es la propia marca o el sello personal del que lo ejecuta, lo cual depende de las actitudes formadas en la persona para llevar a cabo el acto, así como cada persona piensa de manera diferente, los actos violentos también lo son: mientras algunos agreden tímidamente, otros se ensañan sádicamente contra sus víctimas.

Contexto posibilitador. Aquí se trata tanto del contexto mediato (social) e inmediato (situacional) en el que se desenvuelve un individuo, lo cual lo conduce o no a actuar violenta o agresivamente.

Respecto del contexto mediato de la violencia, se considera que la violencia presenta un carácter multidimensional. Los fenómenos que acompañan el comportamiento violento cruzan constantemente las fronteras entre individuo, familia, comunidad y sociedad y, a su vez, las consecuencias abarcan estos ámbitos (Unicef, 2006: 22).

La violencia es el resultado de la acción recíproca de factores individuales, relacionales, comunitarios, sociales y temporales (enfoque ecológico). Perspectiva asumida también por la OMS (2003:13) que señala: la forma en que estos factores están vinculados con la violencia es una de los pasos importantes en el enfoque de salud pública para prevenir la violencia.

Nivel social. En este nivel se estudian los factores macroestructurales que generan un clima de aceptación o rechazo social de la violencia. Al referirse a las condiciones sociales se debe tratar necesariamente de la profunda disparidad socioeconómica en el contexto concreto donde se lleva a cabo esa violencia, diferencia que se ha llegado a considerar natural y a la pobreza y la riqueza como categorías estáticas en la sociedad. Ligada a esta concepción se tiene que hacer referencia a la institucionalización de la violencia, como algo cotidiano, normal, lo que puede llevar a su justificación, acorde con quienes poseen el poder dentro de un país.

Fondo ideológico: justificación de la violencia. Este es el contexto ideológico dentro de la realidad social que se vive en el momento de ejercer el acto violento, lo cual ocasiona que ciertos actos se justifiquen, mientras que otros se condenen. En ese sentido se deben tener en cuenta los aportes de Haber y Seidenberg (1978, citados por Martín-Baró, 2003: 90) que señalan que la justificación de un acto violento depende de cuatro factores, los que se describen a continuación.

- El agente de la acción: El análisis del agente de la acción resulta imprescindible para diferenciar los actos de violencia.

- La víctima: Al igual que los agresores, las víctimas tampoco son iguales. Tanto si la victima proviene de los sectores marginados o de estratos socioeconómicos altos, la magnitud y el impacto de sus problemas en relación a quienes los transmiten guardan relación directa con su situación socioeconómica. 
- La violencia como defensa o agresión: Resulta muy diferente la agresión realizada por un asaltante que la que se utiliza al defenderse violentamente contra él.

- El grado de daño producido: En los actos violentos, mientras mayor sea el grado de daño producido a la víctima, más justificado se debe presentar el acto de violencia.

En relación a la reflexión socio-moral asumimos que en la actualidad el planteamiento gira en torno a que los hechos morales consisten en reglas de conducta sancionadas, siendo la sanción consecuencia del acto, en nuestra sociedad los problemas de corrupción, entre otros, nos conduce a revisar los procesos morales.

Es desde el ámbito de la Psicología que Kohlberg profundiza los trabajos de Piaget, utiliza el termino "concepto moral" como modo de evaluación de lo bueno y de lo recto (Hersh, Reimer y Paolito, 1984). Kohlberg y establece dos factores importantes para el desarrollo cognitivo y la interacción entre iguales y/o con los padres; así como las influencias ambientales y/o culturales. Señala que las etapas son las mismas en los diversos países al margen del contexto cultural.

Kohlberg plantea tres niveles del juicio moral y cada uno de ellos se subdivide en dos etapas o estadios. Los primeros seis estadios se caracterizan por ser invariantes en el orden que alcanzan, ser universales, formar estructuras en conjunto y estar jerárquicamente interrelacionados. Cabe anotar que cada estadio es cualitativamente distinto al anterior y está relacionado con la edad dentro de un agrupamiento general.

En la propuesta de Gibbs (citado por Tapia, Castro \& Monestel, 2007), autor del instrumento que se utiliza en esta investigación, aparece el concepto de desarrollo de la reflexión sociomoral, se refiere a la existencia de una reflexión estructurada, por un lado, por la comprensión de la naturaleza de las relaciones entre las personas y, por otro, como una reflexión estructurada por las transacciones que ayudan a regular, mantener y transformar aquellas relaciones. La adaptación de instrumentos como el "Social Life Questionnaire" o "Sociomoral Reflection Measure Short Form", elaborado por Gibbs, Basinger y Fuller (1992) es otro intento por simplificar la evaluación del desarrollo sociomoral, respecto a la entrevista de Kohlberg.

A nivel internacional se han desarrollado diversas de investigaciones respecto del juicio moral.

Barba y Romo (2005) presentan la evaluación del desarrollo moral de estudiantes de educación superior con base en la teoría de L. Kohlberg. Se analiza su perfil moral según las variables semestre, género, institución, carrera, edad y nivel educativo. Los principales resultados son: el nivel de moralidad predominante es el convencional; las mujeres de una institución privada aventajan a los hombres en la moralidad de principios entre otros.

Romo (2005), en México, investigó el desarrollo del juicio moral y la solución de la crisis de identidad en estudiantes del bachillerato de Aguascalientes. Los principales resultados mostraron que el nivel de razonamiento moral predominante fue el convencional; en moralidad postconvencional se obtuvieron puntuaciones por debajo de otros estudios; la única variable relacionada con el juicio moral fue el género, en favor de las mujeres. 
Retuerto (2004) en Valencia investigó el desarrollo del razonamiento moral, razonamiento moral prosocial y empatía en la adolescencia y juventud. La edad ejerce una influencia significativa sobre el razonamiento moral, sobre el razonamiento prosocial y sobre la empatía de los adolescentes y jóvenes.

Respecto al razonamiento moral, se produce con la edad una transición gradual de una moral convencional a una moral derivada de un código racional, de una moral sociónoma a una moral autónoma. El nivel educativo tiene un efecto significativo sobre el razonamiento moral, sobre el razonamiento prosocial y sobre la empatía de los adolescentes y jóvenes. Tal efecto es similar al producido por la edad. El razonamiento moral, el razonamiento prosocial y la empatía están interrelacionados empíricamente de forma positiva. Se concluye, a su vez, una relación positiva entre los procesos cognitivos y afectivos en el desarrollo moral de la persona.

García y Pérez (2005) realizaron una investigación con 323 estudiantes universitarios. Los resultados obtenidos utilizando el Defining Issues Test-DIT de J. Rest y la Escala de valores (A) de Milton Rokeach, indican que a la convencionalidad moral se asocia una constelación de valores diferente a la que se asocia a la posconvencionalidad moral, en términos estadísticamente significativos. Todo ello permite concluir que el avance del razonamiento moral a través de los estadios de desarrollo moral (estructura del razonamiento moral) se ve acompañado de cambios en la jerarquía de valores asociada (contenido del razonamiento moral), que estructura y contenido del razonamiento moral no son independientes.

Echevarria y Vasco (2006) estudiaron las justificaciones morales sobre lo bueno y lo malo de niñas y niños de contextos considerados violentos y no violentos de una ciudad de la Zona Andina colombiana. Los resultados mostraron que los niños y niñas de ambos contextos comparten algunas preocupaciones morales tales como la preservación de la naturaleza, la protección de la vida y el mantenimiento del orden vigente. También se encontraron diferencias. Entre ellas, en el contexto violento los niños y las niñas enfatizaron la preservación de la propia vida y la lealtad. En el contexto no violento se encontró un marcado énfasis en la perspectiva política con referencia al orden legalmente establecido. Se concluyó que el énfasis de los niños y niñas del contexto violento en la lealtad como principio de acción requiere mayor estudio porque podría configurar un posible tercer principio moral de regulación más allá de los dos principios ya establecidos de la justicia y del cuidado.

Ahora bien, en el Perú, Grimaldo (2009) realizó un estudio que consistió en identificar y comparar el nivel de juicio moral en universitarios, según sexo, rango de edad (entre 16 y 19 años) y facultad, en una muestra conformada por 301 estudiantes del primer ciclo de una universidad pública de Lima, tanto varones como mujeres. Se aplicaron dos instrumentos: El Cuestionario de Reflexión Socio moral y un Cuestionario de Datos Personales. Se concluyó que el grupo se ubica principalmente en el estadio 4, correspondiente al nivel convencional de juicio moral. No se encontraron diferencias significativas en relación con el sexo ni con la facultad de estudio. En cambio, sí se hallaron en lo pertinente a la edad. La juventud activa todos sus mecanismos para que asuma roles y obligaciones nuevas y, por consiguiente, los valores subyacentes (El-Astal, 2008). 
Meneses (2005) realizó un estudio con adolescentes de $4 .{ }^{\text {to }}$ y $5 .^{\text {to }}$ de secundaria que estudiaban en un colegio militarizado con régimen de internado y otro grupo de adolescentes que no estudiaban en Colegio militarizado, ambos grupos fueron evaluados en juicio moral con el Cuestionario de Di BLassio, Pagnani, Pedrabassi y Venuni encontrando que no existen diferencias en el juicio moral entre los adolescentes de ambos grupos.

Altez (2004) presenta el desarrollo del juicio moral en 545 estudiantes universitarias, según edad, ciclo y programa académico. Los resultados mostraron que no existen diferencias significativas entre las edades y ciclos académicos, pero sí existe diferencia entre programas académicos. La muestra se ubica en el Estadio 4 en primer lugar, el estadio 3 en segundo lugar y el estadio 5 en el tercer lugar. Se presenta un baremo basado en la muestra investigada.

En el Perú, Grimaldo (2002) realizó la adaptación del Cuestionario de reflexión socio moral (SROM) de Gibbs y Widaman en 260 estudiantes de $3 .{ }^{\text {er }}, 4 .{ }^{\text {to }}$ y $5 .^{\text {to }}$ de secundaria de un colegio estatal y estudiantes de $1 .{ }^{\text {er }}$ y $3 .{ }^{\text {er }}$ año de la Facultad de Psicología de una universidad estatal. Concluyendo que el Cuestionario de reflexión sociomoral (SROM) constituye un instrumento válido y confiable para medir el desarrollo del juicio moral, además observó una progresión del juicio moral en la medida que aumenta la edad, además no encontró diferencias respecto de la variable sexo.

Grimaldo (1999) estudió las etapas globales de juicio moral en estudiantes de $5 .^{\text {to }}$ año de secundaria, según el nivel socioeconómico y el sexo. Los instrumentos utilizados fueron el Cuestionario de Reflexión Sociomoral-SROM y el Test de Comprensión Lectora de Tapia y Silva (1982). Se concluyó que los estudiantes varones y mujeres de nivel socioeconómico bajo se encuentran principalmente en la etapa 3 de desarrollo de juicio moral; mientras que los estudiantes varones de nivel socioeconómico medio se ubican con mayor frecuencia en la etapa 3(4) y las mujeres del mismo nivel socioeconómico en la etapa 4(3).

\section{OBJETIVOS}

\section{General}

- Relacionar las representaciones sociales sobre la corrupción y la violencia con los niveles de reflexión sociomoral enjóvenes, de ambos sexos, de edades entre 18 a los 25 años de diversas regiones del país.

\section{Específicos}

- Identificar las representaciones sociales sobre la corrupción y la violencia.

- Evaluar los niveles de reflexión sociomoral de los jóvenes urbanos y rurales de diversas regiones del país.

\section{Hipótesis}

Existe relación entre las representaciones sociales de corrupción y violencia con los niveles de reflexion sociomoral de jóvenes de zona rural y urbana de diversas regiones del país. 


\section{MÉTODO}

El presente estudio utilizará el método descriptivo y un diseño cuanti-cualitativo de corte transversal-analítico.

Se evaluará a doscientes jóvenes, de ambos sexos, de edades entre 18 a los 25 años, de diversas regiones del país, comprendiendo departamentos de la costa (Lima, Trujillo, Chiclayo), de la sierra (Arequipa, Huancayo) y de la selva (Amazonas, Tarapoto y Moyobamba, Pucallpa-Ucayali).

\section{Variables}

- Representaciones sociales sobre la violencia y la corrupción.

- Reflexión sociomoral.

- Dado el carácter de los variables a estudiar, se utilizó la técnica psicométrica y los grupos focales desde el enfoque cualitativo.

\section{Instrumentos}

Test de reflexion sociomoral propuesto por Leonard Gordon y Pérez, traducida y adaptada a nuestra realidad por Majluf (1983), citado por Grimaldo (1999). Esta prueba implica el uso de dilemas morales. Las respuestas se tabulan y computan hasta llegar a un puntaje que se traduce en una etapa global del juicio moral, cuyo rango de equivalencia con la escala de niveles de juicio moral de Kohlberg permite ubicar al sujeto en el nivel de madurez que le corresponde. La administración puede ser individual o grupal en un tiempo aprox. de 50 minutos. Se puede tomar a jóvenes desde los catorce años. La confiabilidad del test fue determinada por medio del test-retest, excluyendo la edad, arrojando una correlación de 0.82 a 0.76 . La consistencia interna estimada por el coeficiente de Cronbach fue de 0.84 . La validez concurrente de esta prueba con la medida de SRM (medida de reflexión socio moral) fue de 0,74, p menor 0.01 (Grimaldo, 1999).

Guía de entrevista focal para evaluar las representaciones sociales. En esta guía se incluyen diversas dimensiones tales como: Identificar las representaciones sociales de la corrupción y violencia, justificación, origen, tipificación, el papel de los medios de comunicación, y la prevención. 


\section{RESULTADOS}

\section{Análisis descriptivo}

TABLA 1. Estadios en desarrollo moral

\begin{tabular}{lccc}
\hline Estadio & Denominación & Frecuencia & Porcentaje \\
\hline Estadio 1 & Castigo y orientación a la obediencia & 15 & 9,0 \\
\hline Estadio 2 & Orientación instrumental relativista & 36 & 21,6 \\
\hline Estadio 3 & Orientación a la concordia interpersonal & 115 & 68,9 \\
\hline Estadio 4 & Orientación de la ley y orden & 1 &, 6 \\
\hline & Total & 167 & 100,0 \\
\hline
\end{tabular}

De acuerdo con los resultados de la Tabla 1, el estadio de desarrollo moral más frecuente es el Estadio 3, orientación a la concordia interpersonal con 115 casos que representan el $68,9 \%$ de los sujetos.

TABLA 2. Nivel en desarrollo moral

\begin{tabular}{lcc}
\hline Nivel & Frecuencia & Porcentaje \\
\hline Preconvencional & 51 & 30,5 \\
\hline Convencional & 116 & 69,5 \\
\hline Total & 167 & 100,0 \\
\hline
\end{tabular}

De acuerdo con los resultados de la Tabla 2, el nivel de desarrollo moral más frecuente es el Convencional, con 116 casos que representan el 69,5\% de sujetos.

TABLA 3. Estadios en desarrollo moral según lugar

\begin{tabular}{lcccc}
\hline Lugar & Estadio & Denominación & Frecuencia & Porcentaje \\
\hline Lima & Estadio 1 & Castigo y orientación a la obediencia & 2 & 3,9 \\
& Estadio 2 & Orientación instrumental relativista & 4 & 7,8 \\
& Estadio 3 & Orientación a la concordia interpersonal & 45 & 88,2 \\
\hline \multirow{2}{*}{ Ica } & Estadio 1 & Total & 51 & 100,0 \\
& Estadio 2 & Ccastigo y orientación a la obediencia & 8 & 17,0 \\
& Estadio 3 & Orientación a la concordia interpersonal & 16 & 34,0 \\
& & & 23 & 48,9 \\
\hline Chimbote & Estadio 1 & Castigo y orientación a la obediencia & 47 & 100,0 \\
& Estadio 2 & Orientación instrumental relativista & 3 & 6,0 \\
& Estadio 3 & Orientación a la concordia interpersonal & 36 & 20,0 \\
& Estadio 4 & Orientación de la ley y orden & 1 & 72,0 \\
& & Total & 50 & 2,0 \\
\hline Trujillo & Estadio 1 & castigo y orientación a la obediencia & 2 & 100,0 \\
& Estadio 2 & Orientación instrumental relativista & 6 & 10,5 \\
& Estadio 3 & Orientación a la concordia interpersonal & 11 & 31,6 \\
& & Total & 19 & 57,9 \\
\hline
\end{tabular}


De acuerdo con los resultados de la Tabla 3, el estadio de desarrollo moral más frecuente es el Estadio 3, en todos los lugares observados, siendo en Lima el 88,2\%, en Ica el 48,9\%, en Chimbote 72,0\%, y en Trujillo 57,9\%. Además se observa un caso del Estadio 4 en Chimbote.

TABLA 4. Nivel en desarrollo moral según lugar

\begin{tabular}{llcc}
\hline Lugar & \multicolumn{1}{c}{ Nivel } & Frecuencia & Porcentaje \\
\hline \multirow{4}{*}{ Lima } & Preconvencional & 6 & 11,8 \\
& Convencional & 45 & 88,2 \\
& Total & 51 & 100,0 \\
\hline \multirow{4}{*}{ Ica } & Preconvencional & 24 & 51,1 \\
& Convencional & 23 & 48,9 \\
& Total & 47 & 100,0 \\
\hline Chimbote & Preconvencional & 12 & 24,0 \\
& Convencional & 38 & 76,0 \\
& Total & 50 & 100,0 \\
\hline \multirow{4}{*}{ Trujillo } & Preconvencional & 9 & 47,4 \\
& Convencional & 10 & 52,6 \\
& Total & 19 & 100,0 \\
\hline
\end{tabular}

De acuerdo con los resultados de la Tabla 4, el nivel de desarrollo moral más frecuente es el Convencional, en todos los lugares observados, representando en Lima el 88,2\%, en Chimbote 76,0\%, y en Trujillo 52,6\%. En el caso de Ica el desarrollo moral más frecuente es el Preconvencional 51,1\%.

TABLA 5. Resultados en desarrollo moral

\begin{tabular}{lccc}
\hline & Prob1 & Prob2 & SROM \\
\hline $\mathrm{N}$ & 167 & 167 & 167 \\
Media & 33,24 & 21,69 & 255,39 \\
Desv. típ. & 8,29 & 7,94 & 71,82 \\
\hline
\end{tabular}

${ }^{*}$ Corresponde a nivel convencional.

De acuerdo con la Tabla 5, el promedio en desarrollo moral es 255,39 y la desviación estándar 7,81. Este puntaje promedio corresponde al estadio 3 y nivel Convencional. 
TABLA 6. Resultados en desarrollo moral según lugar

\begin{tabular}{llccc}
\hline Lugar & & Prob1 & Prob2 & SROM \\
\hline \multirow{4}{*}{ Lima } & N. ${ }^{\circ}$ & 51 & 51 & 51 \\
& Media & 35,88 & 24,08 & 280,13 \\
& Desv. típ. & 7,002 & 6,23 & 59,92 \\
\hline \multirow{4}{*}{ Ica } & N. ${ }^{\circ}$ & 47 & 47 & 47 \\
& Media & 30,32 & 18,96 & 227,45 \\
& Desv. típ. & 9,91 & 9,84 & 85,29 \\
\hline \multirow{3}{*}{ Chimbote } & N. ${ }^{\circ}$ & 50 & 50 & 50 \\
& Media & 33,22 & 22,38 & 259,93 \\
& Desv. típ. & 7,14 & 6,79 & 58,59 \\
\hline \multirow{2}{*}{ Trujillo } & N. ${ }^{\circ}$ & 19 & 19 & 19 \\
& Media & 33,42 & 20,21 & 246,15 \\
& Desv. típ. & 8,13 & 7,80 & 75,74 \\
\hline
\end{tabular}

De acuerdo con la Tabla 6, el promedio en desarrollo moral de los sujetos de Lima es 250,13 y la desviación estándar 59,92, en el caso de Ica el promedio es 227,45 y la desviación estándar 85,29, en Chimbote es 259,93 y la desviación estándar 58,59 y en Trujillo es 246,13 y la desviación estándar 75,74. En el caso de Lima y Chimbote estos promedios corresponden al estadio 3 y nivel Convencional, pero en el caso de Ica y Trujillo los promedios corresponden al estadio 2 y nivel Preconvencional.

\section{Contraste de normalidad}

TABLA 7. Prueba de contraste de normalidad

\begin{tabular}{lccc}
\hline \multicolumn{4}{c}{ Kolmogorov-Smirnov } \\
\hline & Estadístico & $\mathrm{gl}$ & Sig. \\
\hline Prob1 &, 100 & 148 &, 001 \\
Prob2 &, 179 & 148 &, 000 \\
SROM &, 173 & 148 &, 000 \\
\hline
\end{tabular}

Seguidamente, en la Tabla 7 se nos muestra las pruebas de normalidad, observando que el nivel de $p$ es no significativo $(p>0,05)$. De este podemos asumir la la variable cuantitativa Desarrollo moral, no presenta distribución normal. 


\section{RESULTADOS}

Resultados referenciales

TABLA 8. Diferencia de medias según lugar

\begin{tabular}{lccc}
\hline & Problema 1 & Problema 2 & SROM \\
\hline Chi-cuadrado & 13,029 & 9,633 & 15,139 \\
gl & 3 & 3 & 3 \\
Sig. asintót. &, 005 &, 022 &, 002 \\
\hline Prueba de Kruskal-Wallis & & \\
\hline
\end{tabular}

Puesto que el nivel crítico es menor que 0,05, en los tres casos, podemos rechazar la hipótesis de igualdad de promedios poblacionales y concluir que las poblaciones comparadas según el Lugar (Lima, Chimbote, Ica y Trujillo) difieren en desarrollo moral (SROM) y en las respuestas al problema 1 y el problema 2 de la prueba SROM.

TABLA 9. Relación entre estadio de desarrollo moral y lugar

\begin{tabular}{lccc}
\hline & Valor & $\mathrm{gl}$ & $\mathrm{p}$ \\
\hline Chi-cuadrado de Pearson & 22,404 & 9 &, 008 \\
Razón de verosimilitudes & 23,143 & 9 &, 006 \\
N. ${ }^{\circ}$ de casos válidos & 167 & & \\
\hline
\end{tabular}

Vemos en la Tabla 9, que el estadístico Chi-cuadrado de Pearson toma un valor de 22,404 el cual, en la distribución $\mathrm{X}^{2}$ con 9 grados de libertad ( $\mathrm{gl}$ ), tiene asociada una probabilidad (p) de 0,008 . Puesto que esta probabilidad (denominada nivel crítico o nivel de significación observado) es muy pequeña, decidimos rechazar la hipótesis de independencia y concluir que las variables estadio de desarrollo moral y lugar están relacionadas.

TABLA 10. Relación entre nivel de desarrollo moral y lugar

\begin{tabular}{lccc}
\hline & Valor & gl & p \\
\hline Chi-cuadrado de Pearson & 21,353 & 3 &, 000 \\
Razón de verosimilitudes & 22,056 & 3 &, 000 \\
N. ${ }^{\circ}$ de casos válidos & 167 & & \\
\hline
\end{tabular}


Vemos en la Tabla 10, que el estadístico Chi-cuadrado de Pearson toma un valor de 21,353, el cual, en la distribución $\mathrm{X}^{2}$ con 3 grados de libertad (gl), tiene asociada una probabilidad (p) de 0,000 . Puesto que esta probabilidad (denominada nivel de significación observado) es muy pequeña, decidimos rechazar la hipótesis de independencia y concluir que las variables nivel de desarrollo moral y lugar están relacionadas.

\section{Resultados cuantitativos}

\section{Con respecto al Problema 1}

\begin{tabular}{lcccccccccc}
\hline & \multicolumn{2}{c}{ Total } & \multicolumn{2}{c}{ Lima } & \multicolumn{2}{c}{ Ica } & Chimbote & \multicolumn{2}{c}{ Trujillo } \\
\hline vida & 44 & $18,5 \%$ & 27 & $27,8 \%$ & 3 & $5,9 \%$ & 9 & $13,6 \%$ & 7 & $\mathbf{2 5 , 9 \%}$ \\
romper ley / principios & 27 & $11,3 \%$ & 12 & $12,4 \%$ & 5 & $9,8 \%$ & 7 & $10,6 \%$ & 4 & $14,8 \%$ \\
préstamo / ahorro & 11 & $4,6 \%$ & 3 & $3,1 \%$ & 2 & $3,9 \%$ & 6 & $9,1 \%$ & 0 & $0,0 \%$ \\
otras actividades & 8 & $3,4 \%$ & 2 & $2,1 \%$ & 3 & $5,9 \%$ & 2 & $3,0 \%$ & 1 & $3,7 \%$ \\
mitad / robar & 1 & $0,4 \%$ & 1 & $1,0 \%$ & 0 & $0,0 \%$ & 0 & $0,0 \%$ & 0 & $0,0 \%$ \\
robar / pago & 4 & $1,7 \%$ & 4 & $4,1 \%$ & 0 & $0,0 \%$ & 0 & $0,0 \%$ & 0 & $0,0 \%$ \\
robar / condena & 13 & $5,5 \%$ & 7 & $7,2 \%$ & 2 & $3,9 \%$ & 4 & $6,1 \%$ & 0 & $0,0 \%$ \\
pagar / interés & 2 & $0,8 \%$ & 2 & $2,1 \%$ & 0 & $0,0 \%$ & 0 & $0,0 \%$ & 0 & $0,0 \%$ \\
ayuda terceros & 17 & $7,1 \%$ & 3 & $3,1 \%$ & 4 & $7,8 \%$ & 7 & $10,6 \%$ & 3 & $11,1 \%$ \\
no solución & 15 & $6,3 \%$ & 4 & $4,1 \%$ & 3 & $5,9 \%$ & 6 & $9,1 \%$ & 2 & $7,4 \%$ \\
otra solución & 27 & $11,3 \%$ & 6 & $6,2 \%$ & 8 & $15,7 \%$ & 9 & $\mathbf{1 3 , 6 \%}$ & 4 & $14,8 \%$ \\
conversar & 4 & $1,7 \%$ & 2 & $2,1 \%$ & 0 & $0,0 \%$ & 1 & $1,5 \%$ & 1 & $3,7 \%$ \\
robar / nuevo problema & 23 & $9,7 \%$ & 7 & $7,2 \%$ & $\mathbf{1 0}$ & $\mathbf{1 9 , 6 \%}$ & 4 & $6,1 \%$ & 2 & $7,4 \%$ \\
sin salida / única solución & 18 & $7,6 \%$ & 3 & $3,1 \%$ & 8 & $15,7 \%$ & 5 & $7,6 \%$ & 2 & $7,4 \%$ \\
último recurso / salida & 5 & $2,1 \%$ & 5 & $5,2 \%$ & 0 & $0,0 \%$ & 0 & $0,0 \%$ & 0 & $0,0 \%$ \\
incomprensión & 1 & $0,4 \%$ & 1 & $1,0 \%$ & 0 & $0,0 \%$ & 0 & $0,0 \%$ & 0 & $0,0 \%$ \\
benevolencia & 2 & $0,8 \%$ & 2 & $2,1 \%$ & 0 & $0,0 \%$ & 0 & $0,0 \%$ & 0 & $0,0 \%$ \\
derecho ganar & 2 & $0,8 \%$ & 1 & $1,0 \%$ & 0 & $0,0 \%$ & 1 & $1,5 \%$ & 0 & $0,0 \%$ \\
confiar Dios & 2 & $0,8 \%$ & 1 & $1,0 \%$ & 0 & $0,0 \%$ & 0 & $0,0 \%$ & 1 & $3,7 \%$ \\
conciencia / justo & 5 & $2,1 \%$ & 2 & $2,1 \%$ & 1 & $2,0 \%$ & 2 & $3,0 \%$ & 0 & $0,0 \%$ \\
avaricia / egoismo & 7 & $2,9 \%$ & 2 & $2,1 \%$ & 2 & $3,9 \%$ & 3 & $4,5 \%$ & 0 & $0,0 \%$ \\
\hline & 238 & & 97 & & 51 & & 66 & & 27 & \\
\hline
\end{tabular}

En general, se considera que robar la medicina se justifica porque hay que preservar la vida (18,5\% de las respuestas). En el caso de Lima y Trujillo, el 27,85 y 25,9\% de respuestas, respectivamente, se orientan en esta dirección. En Ica el robar la medicina crea nuevos problemas $(19,6 \%)$ y en Chimbote considera buscar otras soluciones $(13,6 \%)$. 
Con respecto al Problema 2

\begin{tabular}{lcccccccccc}
\hline & \multicolumn{2}{c}{ Total } & \multicolumn{2}{c}{ Lima } & \multicolumn{2}{c}{ Ica } & \multicolumn{2}{c}{ Chimbote } & \multicolumn{2}{c}{ Trujillo } \\
\hline injusto & 20 & $8,4 \%$ & 6 & $6,0 \%$ & 2 & $4,8 \%$ & 11 & $15,3 \%$ & 2 & $7,1 \%$ \\
dinero/esfuerzo & $\mathbf{6 1}$ & $\mathbf{2 5 , 5 \%}$ & $\mathbf{1 9}$ & $\mathbf{1 9 , 0 \%}$ & 11 & $26,2 \%$ & $\mathbf{2 4}$ & $\mathbf{3 3 , 3 \%}$ & $\mathbf{8}$ & $\mathbf{2 8 , 6 \%}$ \\
padre no cumple & 30 & $12,6 \%$ & 18 & $18,0 \%$ & 2 & $4,8 \%$ & 5 & $6,9 \%$ & 5 & $17,9 \%$ \\
José cumple & 7 & $2,9 \%$ & 7 & $7,0 \%$ & 0 & $0,0 \%$ & 0 & $0,0 \%$ & 0 & $0,0 \%$ \\
respeto/acuerdo/promesa & 47 & $19,7 \%$ & 16 & $16,0 \%$ & $\mathbf{1 8}$ & $\mathbf{4 2 , 9 \%}$ & 11 & $15,3 \%$ & 2 & $7,1 \%$ \\
conversar & 3 & $1,3 \%$ & 2 & $2,0 \%$ & 0 & $0,0 \%$ & 1 & $1,4 \%$ & 0 & $0,0 \%$ \\
diversión/papa & 12 & $5,0 \%$ & 7 & $7,0 \%$ & 0 & $0,0 \%$ & 3 & $4,2 \%$ & 2 & $7,1 \%$ \\
llegar acuerdo & 4 & $1,7 \%$ & 2 & $2,0 \%$ & 0 & $0,0 \%$ & 1 & $1,4 \%$ & 1 & $3,6 \%$ \\
trabajar juntos & 1 & $0,4 \%$ & 1 & $1,0 \%$ & 0 & $0,0 \%$ & 0 & $0,0 \%$ & 0 & $0,0 \%$ \\
obedecer/obligación & 25 & $10,5 \%$ & 8 & $8,0 \%$ & 1 & $2,4 \%$ & 13 & $18,1 \%$ & 4 & $14,3 \%$ \\
reembolso & 5 & $2,1 \%$ & 2 & $2,0 \%$ & 1 & $2,4 \%$ & 1 & $1,4 \%$ & 1 & $3,6 \%$ \\
egoísmo & 5 & $2,1 \%$ & 5 & $5,0 \%$ & 0 & $0,0 \%$ & 0 & $0,0 \%$ & 0 & $0,0 \%$ \\
padre/prestarse otro & 7 & $2,9 \%$ & 4 & $4,0 \%$ & 3 & $7,1 \%$ & 0 & $0,0 \%$ & 0 & $0,0 \%$ \\
padre equivocado/autoritario & 3 & $1,3 \%$ & 2 & $2,0 \%$ & 0 & $0,0 \%$ & 0 & $0,0 \%$ & 1 & $3,6 \%$ \\
imprevisto & 2 & $0,8 \%$ & 1 & $1,0 \%$ & 0 & $0,0 \%$ & 1 & $1,4 \%$ & 0 & $0,0 \%$ \\
padre/ahorro & 5 & $2,1 \%$ & 0 & $0,0 \%$ & 3 & $7,1 \%$ & 1 & $1,4 \%$ & 1 & $3,6 \%$ \\
postergar & 2 & $0,8 \%$ & 0 & $0,0 \%$ & 1 & $2,4 \%$ & 0 & $0,0 \%$ & 1 & $3,6 \%$ \\
\hline & 239 & & 100 & & 42 & & 72 & & 28 & \\
\hline
\end{tabular}

En general, se considera que negar al padre el dinero se justifica porque este es producto del esfuerzo del niño (25,5\% de las respuestas). En el caso de Lima, Chimbote y Trujillo, el $19,0 \%, 33,3 \%$ y 28,6\% de respuestas respectivamente se orientan en esta dirección. En Ica negar el dinero se justifica porque hay que respetar el acuerdo previo $(42,9 \%)$.

\section{DISCUSIÓN}

El presente estudio revela que en la población de jóvenes estudiada existe un desarrollo moral mayormente ubicado en relación a la concordia interpersonal aunque un tercio de la población se ubica en una orientación instrumental relativista y mayormente el nivel de desarrollo moral alcanzado corresponde al convencional, sin embargo un porcentaje considerable que supera el $38 \%$ se ubica a un nivel preconvencional. Es decir, muy pocos se orientan hacia la ley y el orden y hacia un nivel postconvencional.

Respecto a la identificación de la corrupción, vemos que las personas (76\%) las representan como actos ilícitos, inmorales que transgreden las normas sociales, manipulación y abuso de poder. Así mismo, hay quienes las conciben en un nivel más elevado como un problema estructural, es decir que está en la sociedad misma, por tanto como un problema psicosocial, ello se da en un $24 \%$ de las personas participantes en la investigación. En ambos casos la finalidad de la corrupción es el beneficio propio, el cual siempre perjudicará a uno o más personas y/o instituciones. Sin embargo, de acuerdo a lo señalado por los participantes, en condiciones específicas un acto de corrupción puede estar "justificado" de acuerdo al 
fin que se busca con ello, por ejemplo en las situaciones hipotéticas (planteadas por el SROM), así, se considera que robar la medicina se justifica porque hay que preservar la vida (18,5\% de las respuestas). En el caso de Lima y Trujillo, el 27,85 y 25,9\% de respuestas, respectivamente, se orientan en esta dirección. En Ica el robar la medicina crea nuevos problemas $(19,6 \%)$ y en Chimbote considera buscar otras soluciones $(13,6 \%)$.

Por ende, habría cierta disonancia, entre lo que se considera corrupción y ante cierta situación hipotética señalada.

Analizando por poblaciones específicas encontramos que la mayoría se ubica en una orientación instrumental relativista en el caso de Pucallpa y en los demás lugares estudiados (Lima, Ica, Chimbote y Trujillo) se ubican en una orientación hacia la concordia interpersonal. Es decir, encontramos mayores problemas en el desarrollo moral en Pucallpa y donde se aminora el problema es en Lima, pero siempre ubicándose dentro de la orientación hacia la concordia interpersonal. Lo cual nos muestra que es posible intervengan problemas de formación donde determinadas poblaciones no tienen tanto acceso a información y desarrollo de habilidades sociales como existe en urbes como Lima, sin embargo estos recursos y posibilidades no posibilitan el suficiente desarrollo moral. Vemos que la ciudad que le sigue a Lima en nivel de desarrollo es Chimbote, pero también dentro de las limitaciones señaladas.

Así, según lugares, el nivel de desarrollo moral alcanzado más frecuente en las ciudades es el convencional, presentándose con mayor porcentaje en Lima y le siguen Chimbote y Trujillo; sin embargo, Ica y Pucallpa se ubican en un nivel preconvencional que supera ligeramente la mitad de la población.

En general, la población estudiada se ubica en el estadio de orientación a la concordia interpersonal y el nivel convencional. Lo cual asociado a lo anterior, puede estar reflejando que se prioriza la afiliación en las relaciones interpersonales al decidir sobre cuestiones morales o éticas, es más importante el llevarse bien con los otros y adaptarse. Razonándose en un considerable porcentajes poblacional, que los actos de corrupción o inmorales hasta pueden considerarse habilidades, lo cual implica fuertes influencias ideológicas que justifican la corrupción y los actos inmorales. Sin embargo, un significativo porcentaje poblacional trata de mantenerse dentro de lo convencional, pero sin proyectarse mayormente hacia lo postconvencional.

Se encuentra que hay mayor desarrollo moral en el caso de Lima y le siguen Chimbote y Trujillo en ese orden, es decir los dos primeros se ubican en un estadio de orientación hacia la concordia interpersonal y en un nivel convencional; pero las demás ciudades se ubican en una orientación instrumentalista y un nivel preconvencional.

Sin embargo, el análisis estadístico de significatividad nos muestra que la variable desarrollo moral no presenta distribución normal. Lo cual nos llevaría a rechazar la hipótesis de igualdad de promedios poblacionales y concluir que las poblaciones comparadas difieren en desarrollo moral según los instrumentos utilizados. Hay diferencias claras entre ciudades y no se puede generalizar y decir que en el Perú el desarrollo moral se ubica en un determinado nivel.

Más bien sí se puede decir que las variables de estadio de desarrollo moral y lugar están 
relacionadas, esto demandaría profundizar en la investigación de los motivos por los cuales se presentan estas diferencias. Igual sucede con las variables de nivel de desarrollo moral que muestran que están relacionadas con el lugar.

Si se intenta precisar diferencias significativas, se llega a rechazar la hipótesis de igualdad de promedios de los distintos lugares y más bien sí hay claras diferencias significativas. Estas diferencias son a favor de Lima. Lo que nos debe llevar a reflexionar sobre qué posibilidades, recursos y espacios de desarrollo ofrece Lima.

Al analizar sobre cómo se reflexiona sobre problemas morales, encontramos que se justifica cometer actos inmorales en función de preservar la vida, sobre todo en el caso de Lima y Trujillo, que presentan muy similares porcentajes. En caso de Ica se acepta infringir la norma y crea nuevos problemas, en Chimbote mayormente se considera buscar otras soluciones, y en Pucallpa la búsqueda de ayuda de terceros. Aquí habría que preguntarse hasta qué punto influyen limitaciones de habilidades sociales y habilidades para la vida como asertividad, autoestima y habilidad para manejar los conflictos. Asimismo, nos llevaría a indagar sobre la influencia de problemas ideológicos y emocionales relacionados a dependencia, problemas de reflexión moral, disonancia cognitiva.

Lo anterior, corrobora los hallazgos de anteriores investigaciones que indican que en las poblaciones infractoras a las normas y leyes se da menor inteligencia social o menos capacidad para desenvolverse en el ambiente social, es decir esto resalta la importancia de estas habilidades para prevenir infracciones a las normas. Aspectos que debieran precisarse mejor en posteriores investigaciones.

Si vemos los resultados, estos no difieren tampoco de hallazgos de otras investigaciones que relacionan el cometer infracciones con factores como: anonimato, inferioridad, azar, ignorancia, supervivencia, confusión y miedo de la gente, conocimiento y costumbre, deseo de no correr riesgos. Por ello, investigaciones posteriores, deben indagar sobre las relaciones entre reflexión moral y acto moral.

Igualmente, vemos que este estudio no difiere de otros que señalan que en relación a lo moral referido a lo puramente individual parte de la determinación de posibilidades de intervención del control formal, de allí se pasa a la búsqueda de oportunidades favorables. Más aun, si en la sociedad la neutralización no es eficaz, es posible se justifique actos inmorales.

Respecto al papel del grupo en la realización del delito, que parte de una ausencia de motivación para actividades aprobadas socialmente que muestran otras investigaciones, la presente investigación muestra que ya a nivel de la reflexión sociomoral existen condiciones pues no se encuentran en nuestra sociedad actividades alternativas que prevengan realmente la corrupción y la infracción moral.

También este estudio no difiere de otros que indican que los individuos más se preocupan por conseguir la aceptación de quienes ya componen el grupo. Es decir, más les preocupa ser aceptados que si su acto será realmente moral o no.

En esta investigación también se encuentra que las prácticas corruptas son concebidas en términos de intercambios ilegales o ilegítimos al margen de la ley y en perjuicio del bienestar social. Pero en muchos casos se tiene una visión pesimista sobre la posibilidad 
de eliminarlas.

Es decir, sobre el tema de la corrupción está muy extendida la desesperanza aprendida en la población, lo que se relaciona paradójicamente con una limitada reflexión sociomoral.

Estas representaciones están impregnados del sistema de valores sociales y culturales de nuestra sociedad, como ocurre también en otras realidades, llevando implícita o explícitamente una valoración de la situación. Las ideas sobre la corrupción son concebidas como una transgresión ética que atenta contra el bienestar social y tiene un alto contenido valorativo negativo. Los sentimientos de sufrimiento, desconfianza y vergüenza, están presentes en los discursos de los jóvenes, mientras que en otros son resaltados los sentimientos de frustración y desamparo frente a la corrupción. Pero la gran mayoría coincide en la inevitabilidad de la corrupción como algo que cubre toda la sociedad.

Igual como en otras sociedades latinoamericanas, en el Perú se ve a la corrupción como un modo de práctica social instaurada en la cultura social. Hay fuerte censura de los actos corruptos que se ven como muy generalizados, lo que ratifica investigaciones sobre percepción de la corrupción efectuadas por Amnistía Internacional que indican que se percibe que se ha acentuado la corrupción en el país.

La representación social sobre la ética en nuestro país, engloba conceptos cognitivos. actitudes y valores, como muestran investigaciones en otros países latinoamericanos.

Así la reflexión moral y el desarrollo moral están considerablemente influidos por la acción colectiva del grupo, lo que coincide con investigaciones en otras realidades latinoamericanas.

\section{CONCLUSIONES}

1. El cuestionario SROM adaptado presenta validez de constructo y confiabilidad.

2. Se comprueba parte del tercer objetivo referido a la relación significativa entre las representaciones sociales sobre la corrupción con los niveles de reflexión sociomoral. En el caso de la relación entre Representación de violencia y Reflexión sociomoral, no se presenta asociación significativa.

3. La interacción más frecuente considera a la corrupción como una práctica social adaptativa que permite la orientación a la concordia interpersonal.

4. En relación a la prevención de la corrupción y la violencia existe la afirmación de que sí son posibles de prevenir.

5. El estadio de reflexión sociomoral más frecuente está referido a Orientación a la Concordia Interpersonal.

6. El nivel de reflexión sociomoral más frecuente es el Convencional.

7. Se comprobó la existencia de asociación entre el nivel y estadio de reflexión sociomoral y lugar de procedencia.

8. El estadio de reflexión sociomoral más frecuente en Pucallpa es el Estadio 2 y en 
el resto de lugares es el Estadio 3 ocupando diversas posiciones Lima (88,2\%), Ica $(48,9 \%)$, Chimbote $(72,0 \%)$, y Trujillo $(57,9 \%)$.

9. El nivel de reflexión sociomoral más frecuente es el Convencional, en orden descendente serían Lima, Chimbote, y Trujillo. En el caso de Ica y Pucallpa el nivel reflexión sociomoral más frecuente es el Preconvencional.

\section{REFERENCIAS BIBLIOGRÁFICAS}

1. Altez, I. (2003). El juicio moral en estudiantes universitarios. Avances en Psicología: 40 años de Psicología en UNIFE (177-190).

2. Bosh, J. (1998). Para conocer las sectas. Editorial Verbo Divino. Estella.

3. Canepa M. (1993). Esquinas, rincones y pasadizos, bosquejos sobre la juventud peruana. Lima: Ed. CEP.

4. Echebarría y otros (1995). Psicología social de prejuicio y el racismo. Madrid: Centro Estudios R. Areces.

5. Fondo de las Naciones Unidas para la Infancia (Unicef) (2006). La violencia contra niños, niñas y adolescentes. Santo Domingo: Unicef.

6. Freire, Paulo (2005). Pedagogía del oprimido. 55. ${ }^{\text {a }}$ ed. México: Siglo XXI. (Edición original, 1970).

7. Gibbs, J.C., Basinger, K., and Fuller, D. (1992). Moral maturity: Measuring the development of sociomoral reflection. New Jersey: Erlbaum.

8. Gibbs, Arnold, Morgan, Schwatz, Gavaghan \& Tappan (1984). Construction and validation of a multiple choice measure of moral reasoning. Child Development, 55, 527-536.

9. Kohlberg, L. (1984). Essays on Moral Development, Vol. II: The Psychology of Moral Development. San Francisco: Harper and Row,

10. Grimaldo, M. (1999) ¿Hacia donde van nuestro jóvenes? Un estudio de los niveles de juicio moral en estudiantes de $V$ de secundaria de niveles socioeconómicos medio-bajo. Lima: Rastros Gráficos SA USMP.

11. Grimaldo, M. (2002). Adaptación del Cuestionario de Reflexión Sociomoral (SROM) de Gibbs \& Widaman. Lima: Universidad de San Martín de Porres.

12. ILAS (1994). Psicología y violencia política en América Latina. Ponencias de especialistas de América Latina. CESOC: Chile

13. Matín-Baró, I. (1997). Acción e ideología. Psicología Social en Centroamérica $U C A$. El Salvador.

14. Montenegro, E. (1987). Un estudio de juicio moral y el nivel de pensamiento en un grupo de maestros de educación infantil. Tesis de Maestría no publicada. Lima: Universidad Peruana Cayetano Heredia. 
15. Morales y Yubero (1996). Del prejuicio al racismo: perspectivas psicosociales. Univ. Castilla la Mancha Cuenca.

16. Moreno, F. (2001). Un modelo teórico para el estudio de la violencia. Madrid: Universidad Complutense de Madrid (UCM).

17. Moscovici, Mugny y Pérez (1987). La influencia social inconsciente. Estudios Psicología Social Anthropos. Barcelona.

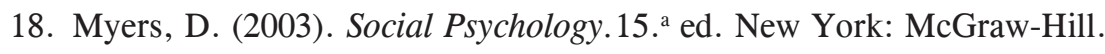

19. Organización Mundial de la Salud (OMS) (2003). Informe mundial sobre la violencia y la salud. Washington, D. C.: OMS.

20. Ortiz, M. y Abad, M. A. (1985) Escala de valores en los autoritarios. Análisis y Modificación de Conducta. Vol 11 N. ${ }^{\circ} 29,345-365$.

21. Pelechano, V. (2001) Autoritarismo, dogmatismo y antiautoritarismo: el cuestionario DOGYANT. Análisis y Modificación de Conducta. Vol. 27, N. ${ }^{\circ}$ 114; 529-580.

22. Peiró, Morales y Fernández Dols (ed.) (2000). Tratado de psicología social. Vol II. Madrid: Síntesis.

23. Raffo, Stronaiuolo, Cristal (2004). Llaki Onqoy, la enfermedad de la tristeza. Lima: Ed. CAPS.

24. Real Academia Española (RAE) (2001). Diccionario de la Lengua Española. 22. ${ }^{\mathrm{a}}$ ed. Madrid: RAE.

25. Rodríguez Carballeira, A. (1992). El lavado de cerebro. Psicología de la persuasión coercitiva. Barcelona: Ed. Boixareu Universitaria.

26. Romo, J. (2005). Desarrollo del juicio moral en bachilleres de Aguascalientes. Revista Mexicana de Investigación Educativa, 24(10), 43-66.

27. Sabucedo, J.M. (1996). Psicología política. Madrid: Síntesis.

28. Theidon, K. (2004). Entre prójimos. Perú: Instituto de Estudios Peruanos (IEP). 